\title{
Malleable parallelism with minimal effort for maximal throughput and maximal hardware load.
}

\author{
F. Spenke* ${ }^{*}$ K. Balzer ${ }^{\dagger}$, S. Frick $\ddagger$, B. Hartke ${ }^{*}$, and \\ J. M. Dieterich ${ }^{+}$ \\ * Institute for Physical Chemistry, \\ Christian-Albrechts-University, \\ Olshausenstr. 40, \\ 24098 Kiel, GERMANY \\ $\dagger$ University Computing Center, \\ Christian-Albrechts-University, \\ Ludewig-Meyn-Str. 4, \\ 24118 Kiel, GERMANY \\ $\ddagger$ Zuse Institute Berlin (ZIB), \\ Takustr. 7, \\ 14195 Berlin, GERMANY \\ + Mechanical and Aerospace Engineering, \\ Princeton University, \\ Princeton, NJ 08544-5263, USA
}




\section{Abstract}

In practice, standard scheduling of parallel computing jobs almost always leaves significant portions of the available hardware unused, even with many jobs still waiting in the queue. The simple reason is that the resource requests of these waiting jobs are fixed and do not match the available, unused resources. However, with alternative but existing and well-established techniques it is possible to achieve a fully automated, adaptive parallelism that does not need pre-set, fixed resources. Here, we demonstrate that such an adaptively parallel program can run productively on a machine that is traditionally considered "full" and thus can indeed fill in all such scheduling gaps, even in real-life situations on large supercomputers in which a fixed-size job could not have started.

\section{keywords}

adaptive parallelism, malleable parallelism, scheduling, genetic algorithms, non-deterministic global optimization 


\section{Introduction}

Traditional job scheduling for high-performance computing (HPC) machines requires fixed, pre-set resources (amount of memory, number of CPUcores or compute nodes, maximum required time) for each job. These settings are provided by the user, and most of them cannot be changed after job submission, neither by the scheduler nor by the user. With a realistic job mix in real-life situations, this leads to a total machine load being substantially smaller than $100 \%$, in at least two generic situations:

1. during standard operation, $N$ compute nodes remain unused because the smallest jobs in the queue requests $M>N$ nodes; hence, typically, an HPC installation is considered "full" already at average loads of $90 \%$;

2. before huge jobs requesting a big portion (50\%-100\%) of the whole machine can run, a correspondingly big portion of the machine has to be drained completely; during this time, no new jobs can start that request more time than the time left until the scheduled start of the big job; typically, this can lead to the load dropping down towards zero, for extended times of several hours.

Idle computing resources are not productive in terms of producing results, but they still cost real money. Electricity consumption of the actual hardware and of the periphery (cooling) may be smaller in idle mode, but is not zero - and should not be zero: In scenario (2) mentioned above, if the huge job finally starts, heat dissipation is instantly required, and then the cooling should already be up and running.

As the size of installations grows, the amount of computing performance left over under these conditions is non-negligible and may in itself be sufficient to run computationally intensive large-scale simulations. Hence, parallel jobs that can be adapted in their resource consumption during runtime can not only use otherwise idle hardware but also allow jobs to start as soon as small amounts of hardware become available for short times, and to grow as soon as more hardware becomes available - in contrast to fixed-size jobs that need to wait until all requested resources are available for the requested time slot. Hence such malleable jobs are highly desirable to fit into this reality, from the perspectives of both the HPC users and the HPC centers.

In a limited way, moldable applications [1-3] already approach some of these goals. For a moldable job, resources are not given as fixed numbers but as ranges or as set of alternatives; actual numbers selected from these possibilities are set at the start of the otherwise traditional, fixed-size application. The only prerequisite is that the application tolerates to be executed with more than one amount of hardware resources. Clearly, under favorable circumstances, this may allow a job to start earlier but still to complete all calculations by running longer. Note that selecting one of several possible resource settings before submitting a job to a queueing system is what most HPC users do manually. In contrast, moldability is about shifting this choice to a slightly later stage (just before execution, and while the job is already in the queue) and/or about automating it, which requires minor or major adjustments in the scheduling/queueing system. In any case, however, once such a job has started, it cannot adapt to changing resource availability at any later point during its runtime — but such changes are happening continuously in any multi-user environment. In contrast, this work addresses malleability or adaptivity, i.e., exactly the feature of adapting to changing resource availability during runtime. 
In the past few years, there has been intensive research on the topic of scheduling malleable parallel applications [4], but actually enabling malleability at the application level has lagged behind, as already diagnosed by other authors [8], and has been confined to the computer-science (CS) literature so far.

Some of these CS studies employed libraries that realize malleability by a brute-force sequence of checkpointing, migrating and restarting the parallel job in question [8,9], implemented on top of MPI-2. Others employ more intricate methods, e.g., the ReShape framework [10,11], also built on-top of MPI-2, or the flexMPI framework [12,13 built on MPI-3, or the proposed ULFM standard API [8] that perhaps will be included in the future MPI-4. Yet other studies 14 17 employed various application management systems (Charmm++, EasyGrid, Nanos ++ ) as additional software layer between the application and the scheduler. In all cases, significant instrumentation of the application source code was needed, to add defined "resize points", to describe data dependencies, to handle data migration, and to achieve communication with the scheduler. Likewise, in most cases the additional software between application and scheduler also had to be modified/extended, to accomodate this scheduler-application communication and to make resizing decisions. The applications employed in these studies ranged from artificial "sleep"-jobs via basic algorithm parts also occuring in real-life programs (e.g., Jacobi or conjugate-gradient algorithms) to small, simplified versions of real-life applications (e.g., simple N-body dynamics). Tests were typically performed on dedicated hardware, against a backdrop of artificial other jobs, with real-life-like characteristics.

One study [11] aimed at applying a ReShape-like approach to the real-life moleculardynamics program LAMMPS [18]. In that case, however, the authors felt the need to limit the necessary source-code changes. Hence, they stepped back from the actual ReShape to employing the checkpoint/restart feature already built into LAMMPS; but even then additional source-code changes were required (including modifications of the input parser).

In contrast to the CS papers listed above, we have employed a truly real-life application, namely our global optimization code OGOLEM [19], which has been thoroughly benchmarked 20 23] and has been used on very diverse, real-life global optimization problems [24 28] However, other than in Ref. [11], we did not resort to a brute-force checkpoint/restart approach to achieve malleability; this feature arises as a side-effect of the remotemethod-invocation-(RMI)-parallelization of OGOLEM which we recently presented [22]. Of course, we originally did have to do some source-code-level integration into OGOLEM as subsequently discussed, but only to introduce the RMI-parallelization itself. Notably, none of the computational kernels needed to be changed and the overall global optimization algorithm remained the same. As already described in Ref. [22], RMI is a simpler and more versatile parallelization basis than MPI, allowing wildly heterogeneous hardware and full fault tolerance $[22]$ without sophisticated add-ons. No additional source-code modifications were necessary to get from this RMI stage to the present malleability demonstration. In particular, and again in contrast to the above CS studies, to achieve this malleability no additional software layer was needed between OGOLEM and the scheduler; the simple reason for this is that in our case no direct communication is needed between the application and the scheduler, whereas in the above-cited studies a direct, two-way communication is essential. In fact, both the hardware fault tolerance demonstrated earlier [22] and the communication-free malleability demonstrated here arise from the same basic feature: In our RMI-based, asynchronous server-client scheme, the server tolerates sudden loss and addition of clients at any point during the overall 
calculation, without the need to insert any resize points or data re-shuffling operations into the application software. Hence, the resources allocated to this whole server-client setup can be set externally by the scheduler, without the need to communicate any resize events to the application level. In fact, in our implementation, the external dependencies are minimal. We almost exclusively rely upon standard components of the Java virtual machine; only the interaction with the scheduler has to be adapted to the actual scheduler present. This clearly maximizes portability. With several application examples on different HPC installations, we show that such transfers between different scheduler/queueing systems are easily possible. Furthermore, we have run our tests and demonstrations not on dedicated HPC hardware sections and against a background-load of artificial jobs, but on standard HPC installations, amidst an everyday job mix generated by all the other users that happened to be present.

Last but not least, our overall aim is different: The CS studies summarized above typically aim at optimizing aims like walltime or hardware energy consumption, for a single malleable job in question, or for several such jobs. Such aims require empirical testing and/or automated decisions involving non-trivial performance forecasts based on collected performance data, in short, they again require considerable additional algorithmic work in an additional software layer. In contrast, we aim at minimizing overall hardware idle time, and at using those otherwise idle resources productively. Importantly, we can achieve this without any additional software, relying solely on a few simple settings and on standard scheduler features.

In the applied scientific computing on today's HPC hardware, malleably or adaptively parallel jobs simply do not occur. Instead, traditional thread-based and plain MPI-1/2based parallelization is the exclusive standard. All jobs are run with fixed resource allocations, pre-set by the users, which leads to the above-mentioned two standard situations, both of which waste considerable HPC resources every day. Additionally, no out-of-the-box fault tolerance is present, resulting in jobs on thousands of nodes being killed if only one of them suffers a hardware failure. Our parallelization strategy described here, and also those mentioned above, can change all that. To promote such paradigm changes in the community of applied scientific computing, we deliberately publish this Article not in a CS journal but in a theoretical chemistry journal.

The paper is organized as follows. Our new malleable parallelization strategy is described in section 2, the main experimental results are in section 3, and the conclusions follow in section 4 .

\section{Malleable parallelization}

For the original, detailed discussion of our RMI-based resilient parallelization, we refer to Ref. [22]. We hence will restrict ourselves in the following to a short discussion of the algorithmic features and details of relevance to this work.

A server process maintains an internal queue of tasks to be accomplished, is responsible to receive intermediate results, and combines them to the final result. This process requires only very little resources, so it is often started on infrastructure nodes. Any number of client processes can attach at any point to the server process to obtain a list of tasks to work on and, upon completing them, return the intermediate results to the server. Server and clients maintain heartbeat connections to assure that client malfunction from, e.g., hardware failures are gracefully handled by the server and 
server shutdowns cause all clients to shutdown reliably on their own. Excellent parallel scalability to 6144 cores for evolutionary algorithms was demonstrated. 22

Obviously, the ability to attach and detach client processes trivially at job runtime lends itself naturally to adapt a job to available computing resources, i.e., be used as a fill-in job. We can hence use scheduling leftovers of ordinary, statically shaped jobs in an efficient manner. As resources become available, clients are started and attach to the server. As resources are needed for static jobs, clients can and will be killed by the queuing system.

To achieve this goal of a complete machine load fill, the fill-in jobs need to be treated differently by the queuing system than ordinary jobs. For an undisturbed workflow of ordinary jobs it is essential to cancel running fill-in jobs to free computing resources. Luckily, this is a standard feature supported by modern scheduling systems. Without excessive, costly, and usually inflexible checkpointing, an ordinary job suffers substantial loss of computing time on a sudden cancellation, so this feature often remains unused. In contrast, in our setup, the master-client dialogue is built on task/result chunks that are communicated frequently and can be controlled in their size and frequency by the user. Additionally, lost computing time due to client cancellation can be rescheduled or, if possible (as in the present evolutionary algorithm (EA) applications), just dropped. Hence, losses upon cancellation can be kept minimal, and the balance between chunk size, communication frequency and computations that possibly have to be re-done can transparently be adapted by the user to an expected interruption frequency scenario.

In contrast to the other approaches mentioned in the introduction, our strategy does not require any direct cooperation between the scheduling system on the one hand and the malleable server-client application described above. In fact, the scheduler remains completely unaware of the presence of malleable jobs; it only sees the presence of higherand lower-priority jobs. The scheduler is free to terminate these lower-priority jobs (RMI clients in our case) at any point without notice, as our approach will internally recover from said termination. Thus, from the viewpoint of the traditional, statically sized, high-priority jobs, there is no difference between free resources and resources used by lower-priority fill-in jobs; the latter are always just as easily and instantly available as the former.

Similarly, more clients can be added to the queue at any point, but again without pre-planning and without app-scheduler communication. If higher-priority jobs leave resources unused, the scheduler will start these clients and they will then productively contribute to the overall RMI server-client system. However, should no unused resources be available currently, the clients simply keep waiting in the queue, without affecting the overall RMI server-client job, except for a reduction in current throughput. This total absence of direct scheduler-app communication not only simplifies the software stack but also significantly reduces error sources arising from mandatory cooperation between application / library and scheduler. The only additional but very small, simple and dependency-free tool needed is a way to re-supply the queue with new waiting client jobs, in a reasonable proportion to the number of clients that transition from waiting to active. This can either be a non-sophisticated shell script that keeps track of the number of clients waiting in the queue, or a queue-script solution that puts a new client into the queue once its own client job gets started.

In order to employ realistic fill-in jobs, we performed global minimum-energy structure optimizations of water clusters with them, using the TIP4P [29] water model, in most 
cases for a cluster size of $\left(\mathrm{H}_{2} \mathrm{O}\right)_{55}$, which is the upper size limit for this system and for this task, in studies published so far [30].

\section{Experimental results}

\subsection{Local hardware}

As a first real-life test, we checked the queue-fill-in capabilities of our RMI setup on the heterogeneous local hardware of the Hartke group. The following machines were involved in the test shown in Fig. 1.

$\begin{array}{cccc}\text { \# nodes } & \text { \# processors } & \text { processor type } & \text { \# cores / processor } \\ 4 & 2 & \text { AMD Opteron 2358 SE } & 4 \\ 2 & 2 & \text { Intel Xeon E5-2680 v4 } & 14 \\ 3 & 2 & \text { Intel Xeon X5675 } & 12 \\ 2 & 4 & \text { AMD Opteron 6172 } & 12 \\ 1 & 2 & \text { Intel Xeon X5355 } & 4 \\ 1 & 2 & \text { AMD Opteron 2427 } & 6 \\ 1 & 2 & \text { Intel Xeon Gold 6154 } & 18\end{array}$

and a login node with 8 processors of type Intel Xeon E5-2620. All nodes run openSuse 42.x. For all calculations a Java Runtime Environment (JRE) version 1.8.0_73 was used. The nodes are connected via $2 \mathrm{x} 1 \mathrm{GBit} / \mathrm{s}$ LACP.

The scheduling system used for batch processing is SLURM. 31 It is configured to distribute the existing resources on a first come, first serve basis. To accommodate the fill-in jobs, a new partition was introduced. Jobs started in this partition are low priority, i.e., they are only eligible to start if no job in another partition can use the existing free resources. Also these low-priority jobs are pre-emptible, i.e., they can be aborted by the scheduler before they reach their currently set walltime. The scheduler regards the resources occupied by jobs in this low-priority, pre-emptible partition as free for jobs in other partitions. If these resources are needed, low-priority jobs are canceled and subsequently requeued by the scheduler.

To test the adaptability of the backfill-jobs against a strongly varying background of normal (fixed-resource) jobs, numerous short-lived jobs were added to the latter category. The normal jobs running during the test consisted of photodynamics simulations with MOPAC and global optimizations with OGOLEM (in shared memory mode). The RMIserver was started on the login node. To variably fill both smaller load gaps but also the maximally available 276 cores with a compromise between high granularity and low job count, 40, 20 and 30 RMI-clients of size 1, 4 and 8 CPU cores were used as backfill-jobs. None of the normal jobs was hindered or affected in any way by the backfill jobs, and no manual guidance of the fill-in was made. Fig. 1 shows a typical resulting breakdown of the total CPU load into these two categories (normal jobs, fill-in jobs). Recording of the CPU load started immediately after the first jobs were submitted and continued for 1000 minutes.

Obviously, despite a strongly varying load of standard jobs, the fill-in automatically tops off the available hardware to maximum load, at all times. 


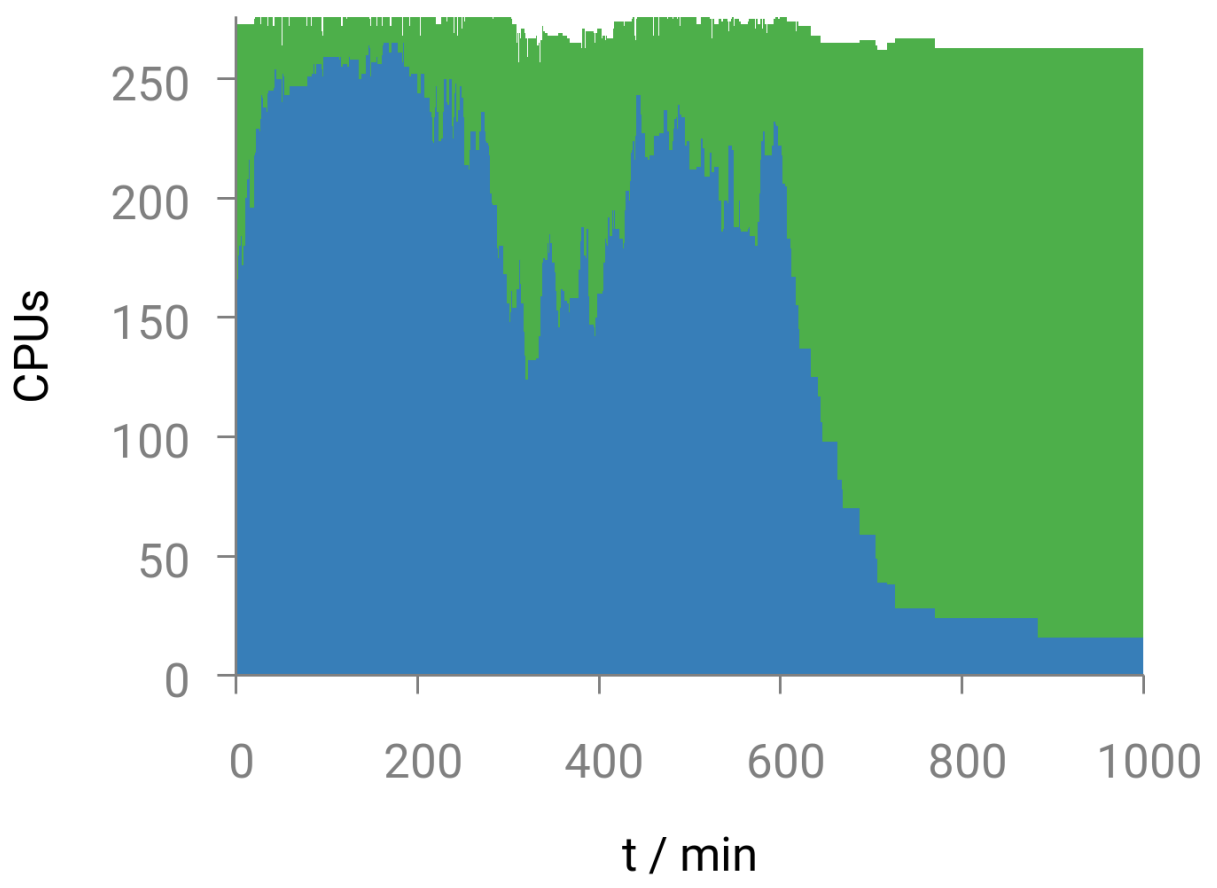

Figure 1: Queue fill-in at the Hartke workgroup computing cluster; normal jobs with fixed resources in blue, RMI-backfill-jobs in green. Note that the normal job set (blue) contains a random admixture of many short-lived jobs, leading to strong, irregular, high-frequency load oscillations. Nevertheless, the RMI-backfill is able to keep the overall load close to $100 \%$ at all times.

\subsection{University computing center}

A similar queue-fill-in was demonstrated on a homogenous subcluster of the university computing center. This cluster consisted of 7 nodes with 40 processors of type Intel Xeon E7-4820 with 10 cores each (i.e., 280 CPUcores overall) and a login node with 8 processors of type Intel Xeon E5-2640. All nodes run CentOS Linux 7.x. For all calculations a JRE version 1.8.0_101 was used. The nodes are connected via InfiniBand.

The used scheduling system is SLURM. Changes to the scheduler are nearly identical to those described in section 3.1. There are again low-priority jobs that are started in a separate partition and can be canceled by the scheduler. In contrast to the above-mentioned scheduler setup, the canceled jobs are not requeued. A fair-share policy is used at this cluster to regulate the start of jobs competing for the same resources. This does not impact the fill-in jobs since these are low-priority and hence do not compete for resources.

The attempt to test the backfill-jobs at this hardware was again accompanied by an artificial background of short-lived jobs in the main partition of the scheduler. The RMI-server was started on the login node. Scripts were used to keep at least 5 backfill-jobs of size 1, 4 and 8 CPU-cores each queued and eligible to start, to be able to fill upcoming load gaps of various sizes with a fairly small number of fill-in jobs. Fig. 2 shows the CPU load during the test. Again the CPU load was recorded for 1000 minutes after the first jobs were submitted. 


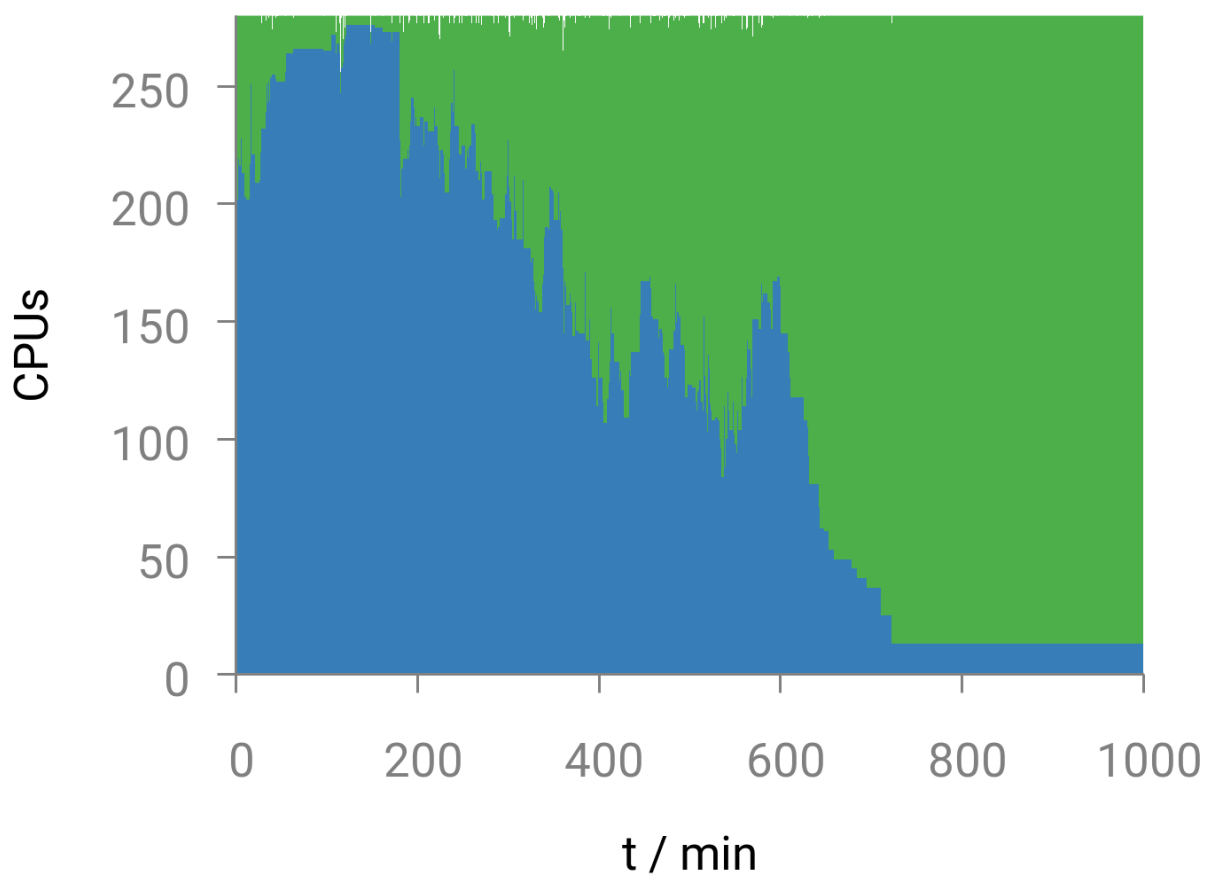

Figure 2: Queue fill-in at the CAUcluster; normal jobs with fixed resources in blue, RMI-backfill-jobs in green.

\subsection{Regional HPC hardware}

A core application case of this work is a demonstration that our flexible RMI parallelization allows us to completely fill even the biggest load gaps on TOP500-class HPC installations, during normal operation and without any impact on other jobs. For such a demonstration, we have chosen the "Konrad" cluster in Berlin, which is part of the North-German Supercomputing Alliance (HLRN) [32] installations in Berlin and Hannover, serving hundreds of HPC users in the North-German states Berlin, Brandenburg, Bremen, Hamburg, Mecklenburg-Vorpommern, Niedersachsen and Schleswig-Holstein. In November 2017, it ranked 182 on the Top500 list. 33

For the demonstration case in this subsection, we used the MPP1 section of "Konrad", a Cray XC30 consisting of 744 Intel Xeon IvyBridge compute nodes. Each node contains 24 CPUcores (in two Intel Xeon IvyBridge E5-2695v2 2.4 GHz processors). Hence, this MPP1 section comprises a maximum of 17,856 CPUcores and has a theoretical peak performance of $342.8 \mathrm{TFlop} / \mathrm{s}$.

As in the tests described above, to allow our fill-in jobs at the HLRN, a new class for pre-emptible, low-priority jobs was specified to the Moab scheduler running there. According to HLRN policies, the number of jobs is restricted via class settings, in our case to 24 simultaneously running jobs.

Additionally, we employed dynamically assigned resources: During a fill-in run we submitted jobs in a per-minute interval that were tailored to fit the available resources at that moment until the next reservation of a regular job.

Independent RMI-client processes were then started on each node. The RMI-server process was started on one of the scheduling nodes of the HLRN.

The dynamically scaled fill-in jobs can easily fill any free nodes. However, some 
restrictions arise due to HLRN user policies and scheduler limitations. The optimal case would be one fill-in job per node. In the event of a newly started ordinary job, exactly the needed number of nodes could be freed and the new job could be started on these. If the cancelled fill-in jobs were bigger than the new regular job, a portion of freed nodes will not be used and needs to be refilled with fill-in jobs.

To adapt to the limited job number according to HLRN user policies, we implemented an additional, automated job monitoring: If this limiting number was to be exceeded, e.g., because another regular job finished execution, the smallest running fill-in job was canceled and a new, larger one was started on the combined free nodes. This once more exploits the high adaptibility built into our concept.

Figure 3 illustrates this fill-in on the HLRN hardware, both during normal operation and during the machine draining period before a huge, machine-wide job starts, i.e., this test covered both scenarios mentioned at the beginning of the introduction. The CPU load was recorded for 1100 minutes in one-minute intervalls, starting after the first batch of jobs was submitted. Additionally, from $t=0$ to $t \approx 200$ minutes, a portion of the nodes was reserved for test jobs by the HLRN, so the maximally fillable node count is slightly lower than during the remaining time. However, our setup is sufficiently flexible to also cope with such changes, without manual interventions.

In contrast to the two test cases described in the previous subsections 3.1 and 3.2 , at the HLRN we had no influence whatsoever on the normal jobs (blue load in Fig. 3). Instead, these jobs had been submitted by other regular HLRN users, as in any other period of normal HLRN operation. Hence, this third test case at HLRN not only demonstrates our adaptive fill-in at a very large HPC center but also under perfectly normal, real-life conditions.

The reactions of our adaptive fill-in to current load levels and their changes are essentially instantaneous, but reaction times of the HLRN queueing system (including simple loadlevel queries, as needed to produce this load figure and to drive the deployment of our fill-in jobs) are not negligible, since high-frequency scheduling/queueing queries were not part of the design specifications when this queueing system was set up several years ago. These delays lead to the jagged appearance of the upper edge of the fill-in load (green area in Fig. 3), occasionally escalating into highly oscillatory load dips (between $t=800$ and $t=900$ minutes) or white "stripes" across the whole (green) fill-in load (between $t=1000$ and $t=1100$ minutes, after the big job finished). These irregularities were much smaller in the other two demonstrations (Figs. 11 and 2). Hence, they are artifacts from a limited scheduler time resolution at HLRN, and do not reflect true deficits of our own fill-in setup. Conversely, to fully exploit these fill-in capabilities, the design specifications of an HPC scheduler installation should include sufficient responsiveness to higher-frequency queries than needed in more traditional HPC scheduling.

With the fill-in jobs displayed in Fig. 3, over $75 \mathrm{M}$ global optimization steps for a $\left(\mathrm{H}_{2} \mathrm{O}\right)_{55}$ cluster in the TIP4P model could be performed and $80 \mathrm{k}$ CPU-hours were used.

Most of these $80 \mathrm{k}$ CPU-hours accrued between $t=800 \mathrm{~min}$. and $t=1000 \mathrm{~min}$., i.e., within only 3 hours and 20 minutes. Nevertheless, this is a substantial HPC resource usage, equivalent to what one full, typical HLRN project uses within one whole week. With conventional parallel (or serial) jobs, these resources would have remained idle, i.e., wasted, as evidenced by the mere existence of all these load gaps and by the obvious fact that normal HLRN jobs were not able to fill them. The HLRN queues were never 


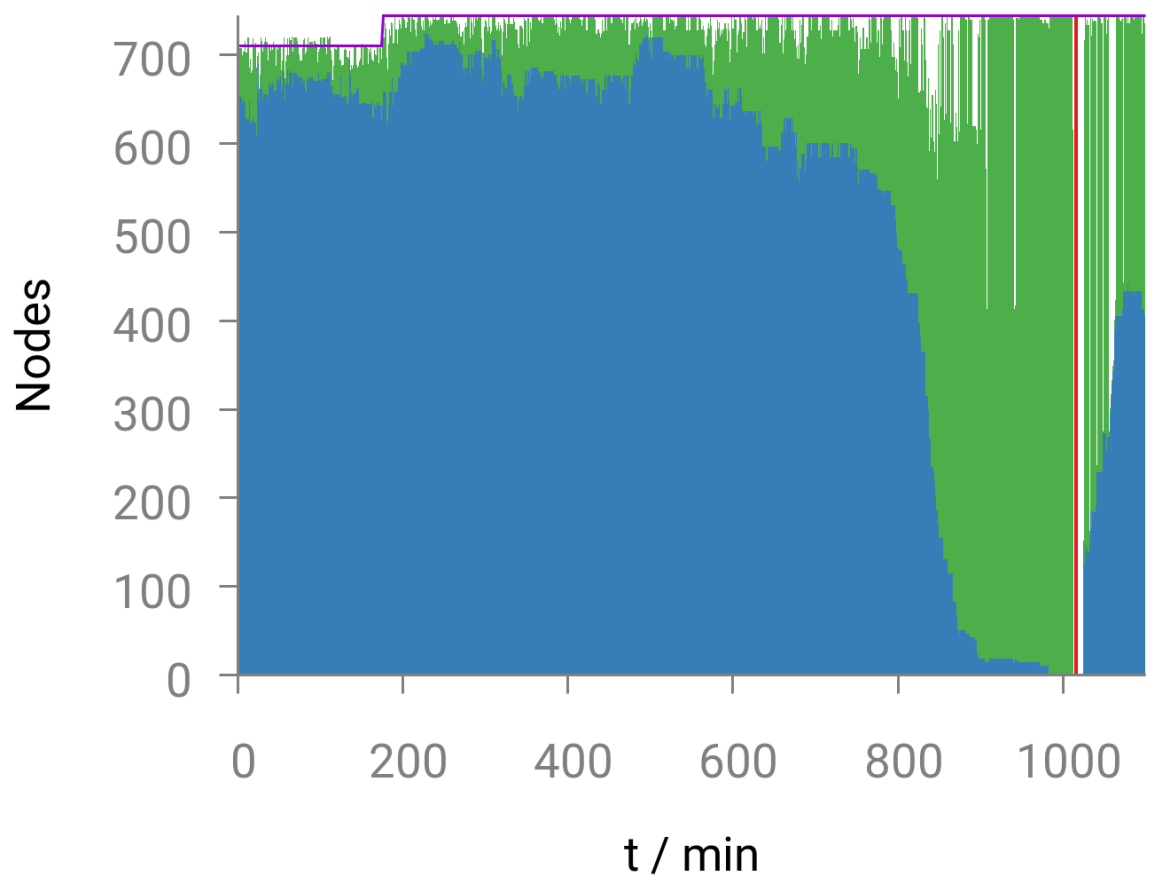

Figure 3: Queue fill-in at the HLRN computing center. A huge job (filling all of the machine) starts at $\mathrm{t}=1010$ minutes (visible only as narrow red line, because it finished again very quickly). In preparation for this event, normal job load (blue) decreases from about $100 \%$ to $0 \%$ between $t=500$ minutes and $t=990$ minutes, in a non-linear and unpredictable manner, since this decrease is not guided externally but arises spontaneously: The remaining time until the pre-set huge job start cannot be used by standard fixed-shape jobs available in the queue. However, our fill-in (green) covers this big gap completely, as it already did during normal operation (before $\mathrm{t}=$ 500 minutes), where the overall load also frequently failed to reach $100 \%$. The blue line indicates the maximally available resources at all times. They were not completely constant: Until $t \approx 200$ minutes, a part of the system was reserved for administrative purposes. Note that 1 node contains 24 CPUcores, hence 744 nodes correspond to 17,856 CPUcores. 
empty during this time, but typically contained 50-100 jobs in a submitted/waiting state, and none of these normal, waiting jobs was in any way hindered by our fill-in jobs, since by construction the former have absolute priority over the latter at all times.

\section{Conclusions}

In summary, we have shown that our highly flexible RMI-parallelization of OGOLEM 22 can indeed be employed to adaptively fill in each and every bit of computing resources that standard parallel jobs under standard scheduling have to leave unused and to use them productively - no matter if these leftovers are small or huge. We have demonstrated this with one and the same program package on three very different computer systems, with significant differences in the queueing/scheduling software and with huge differences in hardware size, ranging from a small, heterogeneous, local computing cluster to a national Top500 supercomputer. To transfer our setup from one of these machines to another one, no changes at all were necessary in our OGOLEM package, and only minor adaptations had to be made in small helper scripts (interacting with the scheduler) and in the scheduler setup (in all cases exclusively exploiting already existing scheduler features). Additionally, on the largest system (HLRN) our demonstration also was a fully real-life case, against a backdrop of standard jobs from many other users, completely beyond our control.

Therefore, with present-day adaptively parallel technologies, even on large-scale HPC installations and in everyday situations, it is now demonstrably possible to avoid all scheduling losses and to achieve a total load level of $100 \%$, at all times, maximally exploiting the available computing resources. Or, in other words, this approach can be used to run HPC jobs with substantial resource needs on HPC machines that are "full" from the perspective of traditional, fixed-size jobs. While achieving $100 \%$ machine load certainly is a desirable goal for any HPC installation, it is not the only one possible with our scheme. With minor additional modifications in the queue configuration or in the mechanism that supplies further client jobs, the maximum allowed load for such an RMI-based server-client job can also be set to arbitrarily smaller load fractions. Again, this can be achieved without any changes in the application software or in the RMI-server-client setup.

Of course, the RMI-parallel model described and demonstrated here is not limited to our OGOLEM code or to GA-like algorithms. The basic ideas that enable both the fail-safe heterogeneity shown in Ref. 22 and the malleability shown here can be transferred to any algorithm that can be set up in a fine-grained server-client model with limited data exchange. Prime examples are all kinds of Monte Carlo (MC) calculations, in chemistry including, e.g., MC-evaluations of thermodynamic quantities, grand-canonical simulations of complex systems, coarse-grained kinetic MC calculations (KMC) of reaction networks, or quantum-MC (QMC) evaluations of the Schrödinger equation, for nuclei or electrons. Another important application area is quantum chromodynamics, where MC-integrations are also heavily used. Such calculations typically take $20 \%$ of all CPU hours used at HLRN. More generally, our paradigm can be easily adopted by any algorithm that generates final results by collecting data from many loosely coupled subtasks, i.e., also by methods like parallel tempering or umbrella sampling molecular dynamics (MD). To enable transfer of the present strategies to even broader classes of algorithms, it may be useful to combine not only thread-based and RMI-based parallelization (as done 
here and in Ref. [22]) but to also include MPI-like parallelization. Future work will include such methodical advances as well as transfers to other types of calculations, as discussed above.

\section{Acknowledgments}

We would like to acknowledge Holger Marten of the Kiel University Computing Center and Thomas Steinke, Christian Schimmel and the "Fachberater" team of the Zuse Institute Berlin (ZIB) / North-German Supercomputing Alliance (HLRN) for allowing us to perform the real-life tests reported here on their machines during normal operation, despite their non-standard queueing/scheduling. Additionally, FS and BH are grateful for a computer time grant which made the big fill-up calculations at HLRN possible, and to Peter Hauschildt (Hamburg Observatory) for submitting huge astrophysics jobs at HLRN, triggering machine drainings that we could then fill up.

JMD wishes to extend his gratitude to Scientific Computing \& Modelling (SCM) who allowed him to pursue these questions in his free time. He also wishes to thank Dean Emily Carter for her current and ongoing support of his other scientific endeavours. $\mathrm{BH}$ is grateful for funding by the Deutsche Forschungsgemeinschaft DFG via project Ha2498/16-1.

\section{References}

[1] W. Cirne and F. Berman. Using moldability to improve the performance of supercomputer jobs. J. Parall. Distrib. Comput., 62:1571-1601, 2002.

[2] S. Srinivasan, S. Krishnamoorthy, and P. Sadayappan. A robust scheduling stratey for moldable scheduling of parallel jobs. In IEEE International Conference on Cluster Computing (CLUSTER 2003), 2003.

[3] N. Rauschmayr and A. Streit. Evaluating moldability of LHCb jobs for multicore job submission. In 15th international symposium on symbolic and numeric algorithms for scientific computing (SYNASC 2013), 2013.

[4] K. Jansen. Scheduling malleable parallel tasks: An asymptotic fully polynomial time approximation scheme. Algorithmica, 39:59-81, 2004.

[5] J. Blazewicz, M. Machowiak, J. Weglarz, M. Y. Kovalyov, and D. Trystram. Scheduling malleable tasks on parallel processors to minimize the makespan. Annals Oper. Res., 129:65-80, 2004.

[6] L. Y. Fan, F. Zhang, G. M. Wang, and Z. Y. Liu. An effective approximation algorithm for the malleable parallel task scheduling problem. J. Paral. Distrib. Comput., 72:693-704, 2012.

[7] Y. J. Cao, H. Y. Sun, D. P. Qian, and W. G. Wu. Scalable hierarchical scheduling for malleable parallel jobs on multiprocessor-based systems. Comput. Syst. Sci. Eng., 29:169-181, 2014. 
[8] P. Lemarinier, K. Hasanov, S. Venugopal, and K. Katrinis. Architecting malleable MPI applications for priority-driven adaptive scheduling. In Proceedings of the 23rd European MPI Users' Group Meeting, EuroMPI 2016, pages 74-81, New York, NY, USA, 2016. ACM.

[9] K. El Maghraoui, T. J. Desell, B. K. Szymanski, and C. A. Varela. Malleable iterative MPI applications. Concurrency Computat.: Pract. Exper., 21:393-413, 2009.

[10] R. Sudarsan and C. J. Ribbens. Design and performance of a scheduling framework for resizable parallel applications. Parallel Comput., 36:48-64, 2010.

[11] R. Sudarsan and C. J. Ribbens. Combining performance and priority for scheduling resizable parallel applications. J. Parallel Distrib. Comput., 87:55-66, 2016.

[12] G. Martín, D. E. Singh, M.-C. Marinescu, and J. Carretero. Enhancing the performance of malleable MPI applications by using performance-aware dynamic reconfiguration. Parallel Comput., 46:60-77, 2015.

[13] M. Rodríguez-Gonzalo, D. E. Singh, J. G. Blas, and J. Carretero. Improving the energy efficiency of MPI applications by means of malleability. In 24th Euromicro International Conference on Parallel, Distributed and Network-Based Processing, 2016.

[14] A. Gupta, B. Acun, O. Sarood, and L. V. Kalé. Towards realizing the potential of malleable jobs. In 21st International Conference on High-Performance Computing (HiPC), 2014.

[15] F. S. Ribeiro, A. P. Nascimento, C. Boeres, V. E. F. Rebello, and A. C. Sena. Autonomic malleability in iterative MPI applications. In 25th International Symposium on Computer Architecture and High-Performance Computing, 2013.

[16] S. Iserte, R. Mayo, E. S. Quintana-Ortí, V. Beltran, and A. J. Pe na. Efficient scalable computing through flexible applications and adaptive workloads. In 46th International Conference on Parallel Processing Workshops, 2017.

[17] S. Prabhakaran, M. Neumann, S. Rinke, F. Wolf, A. Gupta, and L. V. Kalé. A batch system with efficient adaptive scheduling for malleable and evolving applications. In 29th IEEE International Parallel and Distributed Processing Symposium, 2015.

[18] S. Plimpton. Fast parallel algorithms for short-range molecular dynamics. J. Comput. Phys., 117:1-19, 1995.

[19] J. M. Dieterich and B. Hartke. Ogolem: Global cluster structure optimization for arbitrary mixtures of flexible molecules - a multiscaling, object-oriented approach. Mol. Phys., 108:279-291, 2010.

[20] J. M. Dieterich and B. Hartke. Empirical review of standard benchmark functions using evolutionary global optimization. Appl. Math., 3:1552-1564, 2012.

[21] M. Dittner, J. Müller, H. M. Aktulga, and B. Hartke. Efficient global optimization of ReaxFF parameters. J. Comput. Chem., 36:1550-1561, 2015.

[22] J. M. Dieterich and B. Hartke. An error-safe, portable, and efficient evolutionary algorithms implementation with high scalability. J. Chem. Theory Comput., 12:5226, 2016. 
[23] M. Dittner and B. Hartke. Conquering the hard cases of Lennard-Jones clusters with simple recipes. Comp. Theor. Chem., 1107:7-13, 2017.

[24] J. M. Dieterich, U. Gerstel, J.-M. Schröder, and B. Hartke. Aggregation of Kanamycin A: dimer formation with physiological cations. J. Mol. Mod., 17:3195, 2011.

[25] J. M. Dieterich and B. Hartke. Composition-induced structural transitions in mixed Lennard-Jones clusters: global reparametrization and optimization. J. Comput. Chem., 32:1377-1385, 2011.

[26] U. Buck, C. C. Pradzynski, T. Zeuch, J. M. Dieterich, and B.Hartke. A size-resolved perspective of large water clusters. Phys. Chem. Chem. Phys., 16:6859-6871, 2014.

[27] J. Müller and B. Hartke. A ReaxFF reactive force field for disulfide mechanochemistry, fitted to multireference ab-initio data. J. Chem. Theory Comput., 12:3913-3925, 2016.

[28] B. G. del Rio, J. M. Dieterich, and E. A. Carter. Globally optimized local pseudopotentials for orbital-free density functional theory simulations of liquids and solids. J. Chem. Theory Comput., 13:3684-3695, 2017.

[29] W. L. Jorgensen, J. Chandresekhar, J. D. Madura, R. W. Impey, and M. L. Klein. Comparison of simple potential functions for simulating liquid water. J. Chem. Phys., 79:926, 1983.

[30] S. Kazachenko and A. J. Thakkar. Water nanodroplets: Predictions of five model potentials. J. Chem. Phys., 138:194302, 2013.

[31] A. B. Yoo, M. A. Jette, and M. Grondona. SLURM: Simple Linux Utility for Resource Management. In D. Feitelson, L. Rudolph, and U. Schwiegelshohn, editors, Job Scheduling Strategies for Parallel Processing, JSSPP 2003, volume 2862 of Lecture Notes in Computer Science. Springer, Berlin, Heidelberg, 2003.

[32] North-German Supercomputing Alliance (HLRN). https://www.hlrn.de/, accessed: $2017 / 11 / 24$.

[33] Top500 list. https://top500.org/list/2017/11/?page=2, accessed: 2017/11/24. 